\title{
Bilayer Graphene as the Material for Study of the Unconventional Fractional Quantum Hall Effect
}

\author{
Janusz Edward Jacak \\ Additional information is available at the end of the chapter
}

http://dx.doi.org/10.5772/67550

\begin{abstract}
Fractional quantum Hall effect (FQHE) discovered experimentally in 1982 is still mysterious, not fully understood phenomenon. It fundaments are linked with a nontrivial topological effects in 2D space going beyond the standard description of FQHE with local quantum mechanics. The study of integer and fractional QHE in graphene might be helpful in resolution of this fundamental problem in many body quantum physics. FQHE has been observed both in monolayer and bilayer graphene with an exceptional accuracy due to advances in experimental techniques and purity of graphene samples. Recent experimental observations of FQHE in the bilayer graphene reveal different FQHE behavior than in the monolayer samples or in conventional semiconductor $2 \mathrm{D}$ materials. This unexpected phenomena related to Hall physics in the bilayer systems allows to better understand more than 30 years old puzzle of FQHE. In the chapter we will summarize the recent and controversial experimental observations of FQHE in bilayer graphene and describe the topology foundations which may explain the oddness of correlated multiparticle states in the bilayer system. These topological arguments shed also a new light on understanding of heuristic CF concept for FQHE and deeper the topological sense of the famous Laughlin function describing this strongly correlated state.
\end{abstract}

Keywords: monolayer graphene, bilayer graphene, FQHE, hierarchy of filling fractions, braid group for bilayer graphene, correlated state, commensurability

\section{Introduction}

Fractional quantum Hall effect (FQHE) discovered experimentally in 1982 [1] (Nobel Prize 1989 for Tsui and Störmer) is still a mysterious phenomenon robust against its complete 
understanding. The famous Laughlin function [2] proposed for theoretical description of this effect (Nobel Prize 1989 for Laughlin, together with Tsui and Störmer) was the illuminating concept towards explanation of the FQHE. This function was the next start-point for further attempts to understand and describe in detail the experimentally observed FQHE behavior with still growing number of new observations. Despite the breakthrough character of the Laughlin concept, the complete explanation of the FQHE has not been achieved as of yet. The basic problem is linked here with nontrivial topology-type effects in two-dimensional (2D)charged systems exposed to strong perpendicular magnetic field going beyond the standard description of FQHE upon the local quantum mechanics but generating specific long-range correlations in the whole $2 \mathrm{D}$ system.

The Laughlin function has not been derived but it was rather proposed as variational trial multiparticle wavefunction and verified next by numerical exact diagonalization of the interaction of electrons. The archetype for the Laughlin function was the Slater determinant built of zeroth Landau level (LL) single-particle functions. The polynomial part of this Slater determinant has the form of the Vandermonde determinant polynomial and a heuristic change to the Jastrow polynomial results in the Laughlin function. This simple mathematical maneuver has, however, a pronounced physical consequence. The Laughlin function describes the strongly interacting particles, contrary to the Slater function and, moreover, pretty well describes multiparticle correlations of FQHE specific for 2D space topology. The ostensible simplicity of the Laughlin function triggered next efforts to describe FQHE in terms of quasiparticles common in solids, that is, in terms of effective single-particle objects dressed with interaction and defined as the poles of the single-particle-retarded Green function [3]. Nevertheless, in the case of the FQHE, the Coulomb interaction-induced mass operator is discontinuous in 2D at magnetic field presence, and this property precludes any definition of the quasiparticle as dressed electrons with the interaction.

Despite this inability to rigorously define quasiparticles in the FQHE system, a heuristic concept of single-particle picture for this system has been formulated in terms of the so-called composite fermions (CFs) [4]. CF is proposed as complex object consisting of the electron with attached local field flux (even the number of an auxiliary magnetic field flux quanta pinned to the electron). The origin of the auxiliary magnetic field as well as the manner of how the local flux is fixed to a moving electron is not explained, but the CF model works surprisingly well [4]. CFs defined as electrons with fictitious magnetic field flux quanta fixed to them gain the Laughlin function phase shift by the Aharonov-Bohm-type effect when composite particles interchange their position. Note, however, that the Aharonov-Bohm effect is of non-local character. The main advantage of $\mathrm{CF}$ effective single-particle picture resolves itself to the mapping of FQHE onto IQHE in the resultant magnetic field, that is, the true external magnetic field reduced by the averaged field of fluxes pinned to electrons. This mapping allows for the definition of the Jain-like hierarchy of FQHE in partial agreement with experimental data. In view of the above-mentioned discontinuity of the Coulomb interaction mass operator, CFs cannot be named as quasiparticles (moreover, an auxiliary magnetic field fluxes dressing electrons cannot be "produced" by the non-magnetic electric Coulomb repulsion of electrons). One can conclude that CFs describe phenomenologically a different and more fundamental 
property of the system in close relation to the specific topology of $2 \mathrm{D}$ charged system in magnetic field and connected with this topology specific long-range correlation induced by electron repulsion.

In order to decipher this enigmatic character of CFs and the topological correlations in FQHE, the algebraic-topology braid group-based approach has been developed and applied to FQHE [5]. Within this approach, the trajectories of interchanging positions of particles on the 2D plane in the system of $N$ charged particles upon strong perpendicular magnetic field are considered including quantum indistinguishability of identical particles. The geometrically non-equivalent trajectories fall to disjoint classes of nonhomotopic trajectory classes (which cannot be continuously transformed between distinct classes), which form the algebraic group called the braid group. The corresponding quantum multiparticle correlated state is characterized by one-dimensional unitary representation (1DUR) of the braid group for a particular system. For particles in 3D, the braid group of the $N$ particle system is always the $N$-element permutation group (regardless of a charge, interaction, or magnetic field presence). There exist only two 1DURs for any permutation group: $\sigma_{j} \rightarrow\left\{\begin{array}{l}e^{i 0}=1 \\ e^{i \pi}=-1\end{array}\right.$, where $\sigma_{j}, j=1, \ldots, N$, are braids (multiparticle classical trajectories) for exchanges of positions of $j$ th particle with $(j+1)$ th particle, when other particles remain in rest. $1 \mathrm{DUR}=1$ defines bosons and $1 \mathrm{DUR}=-1$ defines fermions in 3D multiparticle systems. For 2D multiparticle systems, the braid group is different than the permutation group [5-7] and 1DURs are different $\sigma_{j} \rightarrow e^{i \alpha}, \alpha \in(-\pi, \pi]$ and define 2D anyons (including 2D fermions for $\alpha=\pi$ and bosons for $\alpha=0$ ).

Nevertheless, in 2D for charged-repulsing electrons in the presence of strong perpendicular magnetic field, the topological situation changes again. For magnetic field strong enough, the only possible on the plane cyclotron classical orbits may be too short to match neighboring particles uniformly distributed on the plane (with classical positions fixed by the repulsion of electrons). Too short braids must be thus removed from the braid group. The remaining braids form the subgroup of the former group called the cyclotronic braid subgroup. The cyclotronic subgroup collects only those braids which can match neighboring particles.

To clarify the structure of the cyclotronic braid subgroups, let us note that in the presence of strong magnetic field the $2 \mathrm{D}$ braids must be built from pieces of cyclotron orbits and this orbit must precisely fit to interparticle spacing. Otherwise, the definition of braids is precluded. Hence, the commensurability of planar braids with particle spacing is an unavoidable condition to define the braid group and establish the quantum statistics (by 1DURs of the braid group). The quantum statistics is a necessary prerequisite to multiparticle-correlated state. Therefore, the commensurability condition determines the magnetic field strength (or equivalently, Landau level-filling rate) at which correlated state can be formed. The archetype of the commensurability in 2D-charged $N$ electron system is the exact fitting of cyclotron orbits of interacting 2D electrons to inter-electron spacing, which happens at completely filled lowest Landau level (LLL), that is, at filling factor $v=\frac{N}{N_{0}}=1$, where $N_{0}=\frac{B S e}{h c}$ is the LL degeneracy $\left(\frac{h c}{e}\right.$ is the magnetic field quantum). In other words, the condition $v=1$ is equivalent with the commensurability condition $\frac{S}{N}=\frac{S}{N_{0}}=\frac{h c}{e B^{\prime}}$, that is, the size of cyclotronic orbit in the LLL at $v=1$ 
fits with particle spacing $\frac{S}{N}\left(S\right.$ is the $2 \mathrm{D}$ plane size for the system; in the thermodynamic limit $\frac{S}{N}$ is constant even if $S$ and $N$ tend to infinity). For $v=1$, the braid group can be thus established and the correlated state manifest itself as the IQHE.

For stronger magnetic fields, $v<1$ and the commensurability fails. It means that ordinary cyclotron orbits $\frac{h c}{e B}$ are too short and the corresponding braids, $\sigma_{j}$, must be removed from the braid group as unavailable ones. In the braid group are, however, braids $\sigma_{j}^{q}$, which for $q$ odd integer similarly as $\sigma_{j}$ define exchanges of $j$ th and $(j+1)$ th particles. In distinction to $\sigma_{j}$, the braids $\sigma_{j}^{q}$ realize exchange with additional $\frac{q-1}{2}$ loops [5]. This additional loops "take away" $\frac{q-1}{2}$ flux quanta. This is the origin of the auxiliary flux tubes pinned to CFs. In $2 \mathrm{D}$, the external field flux per particle $\frac{B S}{N}$ is thus reduced by $\frac{q-1}{2} N$ similarly as in the CF concept. Braids $\sigma_{j}^{q}$ define exchanges along multiloop cyclotron 2D orbits. Exclusively in 2D multiloop cyclotron orbits share the same external field flux per particle and per single loop falls only some fraction of $\frac{B S}{N}$ (noticeably, in contrary to 3D case, when each scroll of spiral adds a new field piercing by the field $B$ ). Hence, in $2 \mathrm{D}$, the multiloop cyclotron orbits related to $\sigma_{j}^{q}$ have larger size and $\sigma_{j}^{q}$ can reach particles out of reach for single-loop braids $\sigma_{j}$. The braid subgroup generated by $\sigma_{j}^{q}, j=1, \ldots, N$ is the proper braid group for $v<1$ provided that the new commensurability condition holds: $\frac{S}{N}=\frac{q B S e}{h c}$, where the r.h.s of this condition expresses the $q$ times larger range of multilooped cyclotron orbit in 2D. From this commensurability condition, it follows the relation: $v=\frac{N}{N_{0}}=\frac{1}{q}$ displaying the main line of LLL fillings for FQHE (described by the Laughlin function with $q$ th order of the Jastrow polynomial). The braid group commensurability is thus the kernel of the Laughlin function derivationany multiparticle ground-state wave function of the correlated 2D N-charged-interacting particles (electrons) must transform according to 1DUR of the related cyclotron braid group [6, 8, 9]. This feature together with requirement that the multiparticle function in the LLL must be holomorphic function of $z_{1}, \ldots, z_{N}\left(z_{j}=x_{j}+i y_{j}-\right.$ complex coordinate on the plane of $j$ th particle) - uniquely results in the form of the Jastrow polynomial $\prod_{i>j}^{N}\left(z_{i}-z_{j}\right)^{q}$ (multiplied by a factor independent of particle interchanges - in the Laughlin function $e^{-\sum_{i=1}^{N}\left|z_{i}\right|^{2} / 4 l_{B}^{2}}\left(l_{B}\right.$ is the magnetic length). The latter term is common for all states from the LLL, thus the derivation of the Laughlin function resolves itself to the determination of the uniform with respect to all particles polynomial factor, which can be deduced according to 1DUR of related cyclotronic braid group as described above.

\section{FQHE in graphene}

Graphene is ideally two-dimensional but with some specific modification of LL structure due to pseudo-relativistic band structure of this gap-less semiconductor. Thus, examining FQHE in graphene is especially challenging. The specific for graphene electronic dynamics is referred to the Dirac point in all corners of the hexagonal first Brillouin zone. This specific pseudo-relativistic local band structure does not influence, however, the topology constraints imposed on 
trajectories and the FQHE has been observed in graphene. Easy methods for the manufacturing of monolayer and bilayer graphene ought to be experimentally noticeable. And indeed, several recent observations of FQHE in bilayer graphene [10-14] demonstrate strong distinctions in comparison to FQHE hierarchy in monolayer graphene [15-18].

The observed FQHE phenomena in bilayer graphene supply an opportunity to verify the cyclotron braid group commensurability approach because the topology of double sheet in bilayer graphene causes different commensurability conditions in comparison to the monolayer case. This will result in specific hierarchy of filling rates for FQHE in bilayer graphene not observed in monolayer graphene and in conventional semiconductor 2D systems.

In the present chapter, we summarize the recent and controversial experimental observations of FQHE in bilayer graphene and describe the topology foundations, which may explain the oddness of correlated multiparticle states for the bilayer system. The significance of such a discussion consists in experimental confirmation of new topological-based theory of FQHE going beyond the phenomenological model of CFs.

Advances in experiment allow now for measurements of longitudinal and transversal resistivity in Hall configurations in various graphene sample arrangements. The great progress in experiment has been achieved by the utilization of measurement technique in the so-called suspended graphene, where small scraping of the graphene sheet has been hanged in free air without any contact with substratum and thus without disturbing phonons in crystals and therefore with extremely high mobility triggering FQHE organization in relatively higher temperatures. It is possible to suspend both monolayer and bilayer sheets. Another experimental achievement is the utilization of homogeneous with graphene crystal substrate of boron nitride $(\mathrm{BN})$ crystal similar as graphene hexagonal surface lattice geometry. The application of BN substrate to support graphene layer also allows for the observation of FQHE in higher temperatures because of reducing stress and perturbations caused by lattice mismatching. The new data for fractional quantum Hall effect both in suspended graphene scrapings $[10-14,17,18]$ and in graphene samples on crystalline substrate of boron nitride $[15,16]$ have been recently collected.

The specific structure of Landau levels (LLs) in graphene leads to distinct than in conventional semiconductor 2DEG scheme for integer quantum Hall effect in graphene referred to as "relativistic" of its version [19]. The graphene planar sheet has a regular crystalline structure with two equivalent sublattices (two carbon atoms in the Bravais cell). This feature together with vanishing of the forbidden semiconductor gap on corners (two of them-non-equivalent) of hexagonal Brillouin zone, with cone-like shape of local valence and conduction bands meeting at these points (the so-called the Dirac points), results in the fourfold spin-valley degeneracy of LLs. Moreover, for Dirac points in graphene, the specific Berry phase-like shift for chiral 2D carriers additionally modifies the LL spectrum, which finally results in $v=4\left(n+\frac{1}{2}\right)$ series for fillings at which IQHE plateaus occur in graphene. These plateaus in the monolayer graphene occur at the centers of the consecutive LLs (not at completely filled LLs) [20]. In bilayer graphene the extra degeneracy of $n=0$ and $n=1$ LLs shifts IQHE plateau positions to edges of LLs, which in bilayer graphene are also fourfold-degenerated except for 
the eightfold-degenerated LLL [19, 20]. For both monolayer and bilayer graphene, FQHE hierarchy of fillings is also observed. In the case of monolayer graphene, the filling fractions for FQHE are observed in six first subbands of LLs with $n=0$ and $n=1$ in Refs. [15-18], which reproduce similar hierarchy known from observations in conventional semiconductors 2DEG. Especially interesting is, however, an observation of unusual even denominator fillings for FQHE in bilayer graphene including the most pronounced feature at $v=-\frac{1}{2}[10]$, which does not find counterpart in monolayer system. This state cannot be also explained upon the CF approach, as this approach suggests the Hall metal state at $\pm \frac{1}{2}$ filling ratio.

In the present paper, we propose the explanation of this exotic, beyond $\mathrm{CF}$, state in bilayer graphene by the topological commensurability approach [6, 21 22]. In this braid group-based approach, we explain the structure of fractional fillings of LL subbands beyond the CF model and explain the reason for the failure of CF model in bilayer graphene. The topological braid group approach formerly developed in $[23,24]$ gives the hierarchy of FQHE in agreement with the available experimental data for monolayer and bilayer graphene in contrary to CF model. It is worth emphasizing the topological explanation of the even denominator-filling ratios for FQHE in bilayer graphene, including $v=-\frac{1}{2}$.

\subsection{Commensurability condition}

Let us briefly recall the concept of the commensurability of cyclotron trajectories with interparticle spacing in 2D-charged system at strong magnetic field within the braid group approach to multiparticle systems at magnetic field presence. It must be emphasized that for FQHE formation, the interaction of electrons is essential similarly as of any other correlated state. The strong Coulomb repulsion of electrons determines the steady uniform distribution of 2D electrons - in the form of triangle Wigner crystal lattice as the classical lowest energy state. Such a distribution of electrons rigidly fixed (in classical sense) by interaction is the starting point for quantization in terms of Feynman path integral including summation on braid group elements assigned with appropriate unitary weight supplied by 1DUR of the braid group [5]. The latter is necessary in the case of multiparticle system as to any path in the multiparticle configuration space an arbitrary closed loop from the braid group can be attached and the resulting paths with such loops fall into geometrically disjoint sectors (topologically non-equivalent) of the path space. This not-continuous structure of the path space, enumerated by the braid group, forces the additional sum over the braid group elements in the general path integration. Each component to this sum must enter the sum with some unitary factor (due to causality condition) and the collection of these factors for all braid group elements establishes its 1DUR. This explains the central role of 1DURs of the braid group for the definition of quantum statistics of multiparticle system. Each different 1DUR defines different sorts of quantum particles corresponding to the same classical ones $[6,9]$. On the other hand, the collective behavior of a quantum multiparticle state must be assigned by the statistics phase shift acquired by the multiparticle wave function if one considers position exchanges of particle pairs [6,9]. The exchange of particles is understood as the exchange of the position arguments of the multiparticle wave function, and these exchanges are defined by the braid group elements. Hence, the determination of the braid group for the multiparticle system is an unavoidable prerequisite for any correlated state. 
The braid group is the first homotopy group of the multiparticle configuration space and describes all possible particle exchanges including quantum indistinguishability of identical particles. This group is denoted as $\pi_{1}(\Phi)=\pi_{1}\left(\left(M^{N}-\Delta\right) / S_{N}\right)$, where $M^{N}$ is the $N$-fold normal product of the manifold $M=R^{2}$ in the case of planar Hall system, $\Delta$ is the diagonal point set in this product (when coordinates of two or more particles coincide, subtracted in order to assure conservation of the number of particles), and $S_{N}$ is the permutation group of $N$ elements [7]. The quotient structure of the configuration space $\Phi=\left(M^{N}-\Delta\right) / S_{N}$ is caused by the indistinguishability of quantum identical particles. The braid group $\pi_{1}(\Phi)$ is a topological object collecting all classes of nonhomotopic trajectory loops in the configuration space $\Phi$, where points which differ only by enumeration of particles are unified. The braid group does not reflect the dynamic details of the system but rather identifies only the topology restrictions imposed on interparticle trajectories conditioned by the geometry-type global features, like the dimension of the manifold $M$.

As mentioned above, the Coulomb repulsion of electrons on the plane is a central prerequisite for the braid group definition at magnetic field presence, because the interparticle separation rigidly fixed by the Coulomb repulsion (in the classical equilibrium state) must interfere with the planar cyclotron orbits, discriminating in this way possible correlation types, by the commensurability condition.

For the same classical particles, the quantumly different particles (as, e.g., bosons and fermions in 3D or anyons in 2D) can be defined as assigned by distinct 1DURs of the related braid group. At the magnetic field presence for 2D-charged particles (as in quantum Hall configuration), there are, however, also other important consequences of 2D topology beyond solely 1DURs of the braid group. For the magnetic field strong enough that the classical cyclotron orbits are shorter in comparison to the interparticle separation on the plane, the braid group must be modified itself. The classical trajectories of charged particles obligatory defined by cyclotron orbits at the presence of a magnetic field allow for braid exchanges only when the size of the cyclotron orbit fits accurately to the separation between particles, as is illustrated in Figure 1 (the separation between particles on the plane is fixed by the Coulomb interaction). It
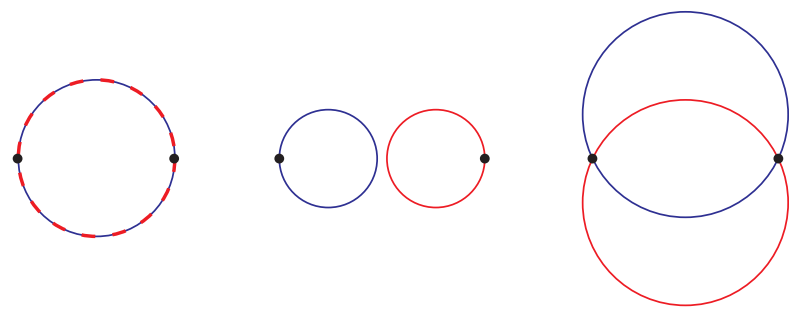

Figure 1. Pictorial illustration of the commensurability (left) between the cyclotron orbits (schematically drawn as circular ones, although at the interaction presence they might be deformed) and the interparticle spacing. This commensurability (also needed at any deformation of the cyclotron orbit by the interaction) satisfies the topology requirements unavoidably needed for the braid interchange implementation in the uniformly distributed particles on the plane with (rigidly fixed particle separation by the electric repulsion). In the case of stronger magnetic fields resulting in shorter cyclotron orbits, particles cannot be matched (center). Similarly for larger cyclotron orbits (at the lower magnitude of the magnetic field), interchanges also cannot be arranged as the interparticle spacing cannot be conserved during exchanges (right). 
is easy to notice that the geometrical planar commensurability between the cyclotron orbit and the interparticle spacing is an unavoidable requirement needed for the definition (implementation) of the generators $\sigma_{j}$ of the braid group. The generators $\sigma_{j}$ describe exchanges of $j$ th and $(j+1)$ th particles. Otherwise, when the cyclotron orbits are incommensurate with the interparticle spacing on the plane, then cyclotron orbits cannot match neighboring particles, and the classical trajectories describing generators $\sigma_{j}$ do not exist in this case-thus the braid group generators $\sigma_{j}$ cannot be implemented. It means that the quantum statistics cannot be defined in this case and any correlated multiparticle quantum state cannot be organized. The possibility to implement the braid group selects thus the magnetic field strengths (or equivalently, filling rates of the LLL) at which the correlated state (FQHE) can be organized. If the braid group cannot be implemented, none of the correlated state is possible at corresponding filling factor. In this way, one can determine the hierarchy of filling rates for FQHE by analyzing the corresponding braid groups. Remarkably, the result fully agrees with all current experimental data for FQHE in any observable Hall systems.

Though the generators $\sigma_{j}$ of the braid group cannot be implemented when the cyclotron orbits are shorter than interparticle separation, there exist other braids, which in $2 \mathrm{D}$ do fit to interparticle separations. Exclusively in 2D, the multilooped cyclotron orbits have larger size, which allows the braids $\sigma_{j}^{q}$ ( $q$ odd positive integer) to fit to interparticle separation. This opportunity happens, however, at some "magic" fractional fillings of the LLL-the same ones at which FQHE is observed. The reason for the enhancement of the size of planar multilooped orbits (and the braid $\sigma_{j}^{q}$ ) is linked with the same surface spanned by 2D orbits regardless of its multilooped character (in opposition to 3D case when each additional loop of the spiral adds an additional surface portion spanned by this loop-in 2D spiral does not exist, however). The external field flux passing through the 2D multilooped orbit must be thus divided among all loops. Hence, the portion of the flux per each loop diminishes, which subsequently results in loop size growth. This is illustrated in Figure 2.

In Figure 2 (left), the scheme of the cyclotron orbit at magnetic field $B$ is shown as accommodated to the quantum of the magnetic field flux, that is, $B A=\frac{h c}{e}$. This is the definition of the cyclotron orbit size $A$. For interacting 2D electrons distributed in the Wigner crystal form, the cyclotron orbits are not circular but their surface $A$ fits exactly to the interparticle separation in the case of the completely filled LLL, $\frac{S}{N}, S$-the sample area, $N$-the number of particles. If only single-looped orbits are considered, then at, for example, $q=3$-times larger field, $3 B$, the cyclotron orbit accommodated again to the flux quantum is too short in comparison to the
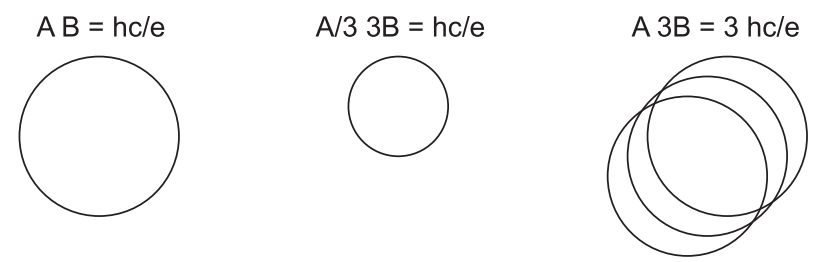

Figure 2. Schematic illustration of cyclotron orbit enhancement in 2D due to multiloop trajectory structure (third dimension added for visual clarity). 
interparticle separation $\frac{S}{N}-$ as is sketched in the central panel of Figure 2. However, in the case when three-loop orbits are considered (note that such orbits are present in the braid group generated by $\sigma_{j}$ generators), then exclusively in 2D space, the external flux $3 B A$ passing through this orbit must be divided among three loops. In the case of the uniform division (when all loops have the same surface $A$ ) per each loop falls a $B A$ fraction of the total $3 B A$ flux. Therefore, one can notice that each loop in this situation, as usual accommodated to the flux quantum $\frac{h c}{e}$, has the orbit with the surface $A$ (the same surface $A$ as for the single-looped orbit at three times weaker magnetic field). Three loops result together in the total flux $3 B A$ per particle, as needed for considered stronger field $3 B-$ cf. Figure 2 (right). It means that the three-loop orbits surprisingly fit to the interparticle separation $\frac{S}{N}=A$.

Because the braid group generator must be defined by half of the cyclotron orbit (cf. Figure 3), thus the braid with one additional loop corresponds to the cyclotron orbits with three loopssuch a generator has the form $\sigma_{j}^{3}$. The group generated by $\sigma_{j}^{3}, j=1, \ldots, N-1$ (new elementary braid exchanges) is obviously the subgroup of the original braid group because its generators $\sigma_{i}^{3}$ are built from original group generators $\sigma_{j}$. This subgroup is called the cyclotron braid subgroup. It is clear that 1DURs of this subgroup define statistics of 2D-charged particles at sufficiently strong magnetic fields, that is, at fields corresponding to the fractional fillings of the LLL provided that the commensurability condition is fulfilled (in the presented example, for $v=\frac{1}{3}$ ). The generalization to more loops attached to the braid generator one by one results

single exchange - braids

a)

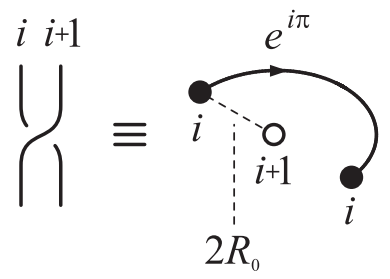

b) $i \quad i+1$

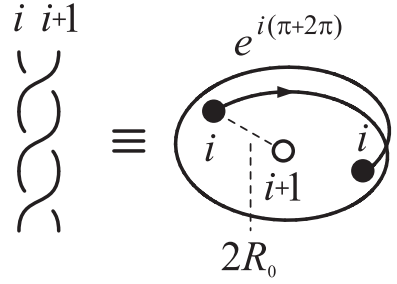

double exchange - cyclotron orbits
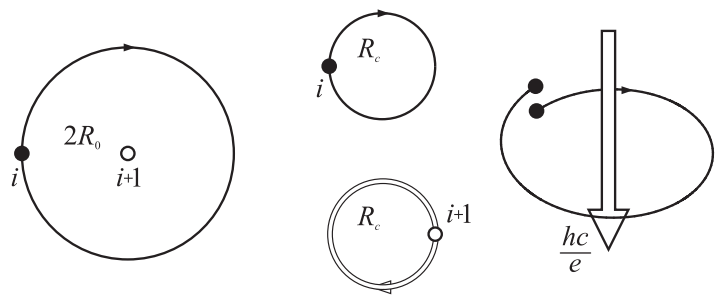

$R_{c}=R_{0}$
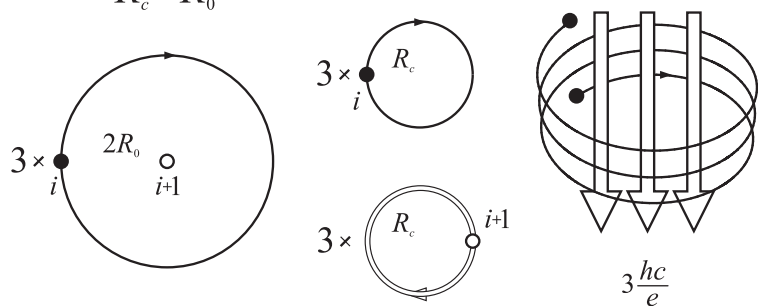

Figure 3. (a) A geometrical presentation of the braid generator $\sigma_{i}-$ it corresponds to an ordinary single exchange of neighboring particles, $i$ th and $(i+1)$ th (left); the cyclotron orbit which realizes this braid exchange at magnetic field presence at $v=1$-drawn in the relative view (right), it is visible that the closed cyclotron orbit corresponds to the double exchange of particles matched by single-looped cyclotron trajectories for $v=1$, as indicated in (a) by $R_{c}=R_{o}$. (b) The threelooped braid generator $\sigma_{i}^{3}$ must be considered for $v=\frac{1}{3}$ to match neighboring particles at three times stronger magnetic field; the additional loop is needed in this case to enhance an effective cyclotron radius, and again in (b) $R_{c}=R_{o}\left(R_{c}-\right.$ cyclotron radius, $2 R_{o}$ - particle separation, i.e., $\left.\pi R_{c}^{2}=\frac{h c}{e B^{\prime}}, \pi R_{o}^{2}=\frac{s}{N}\right)$. 
in double increase of loop number in multilooped cyclotron orbits and thereby in fractions $v=\frac{1}{q}, q$-odd integer. This approach can be easy generalized to higher LLs also in exact agreement with the experimental observations [22].

This generalization to higher LLs resolves itself to the observation that for the filling fractions corresponding to the commensurability condition written as $x \frac{S}{N}=A$ with $x$-positive integer, the cyclotron orbits also fit to equidistantly separated particles, though not to just any but rather to every $x$ th particle. Thus, the commensurability condition $x \frac{S}{N}=\frac{S}{N / x}$ also allows for the definition of the generators $\sigma_{j}$ in the form of ordinary single-looped braids (linking every $x$ th particles, however). This happens for the completely filled higher LLs. The resulting statistics (expressed by 1DUR of these braid groups with longer braids for $x>1$ ) is the same as for IQHE (the longer braids are also single-looped as for IQHE at $v=1$ ). Remarkably, such an opportunity for commensurability happens in higher LLs not only when they are completely filled but also at some fractional fillings, which is illustrated in Ref. [22] in satisfactory good correspondence with the experimental observations available now up to the third LL for 2DEG in conventional semiconductor Hall systems [25-27]. It must be emphasized, however, that the described above situation of too large cyclotron orbits in comparison to the particle separation (and fitting to every $x$ th particle with $x>1$ ) may happen exclusively in such LL subbands for which the commensurability condition $x \frac{S}{N}=A(x-$ integer $)$ is admitted to be fulfilled. Apparently, such a situation may happen only for $n \geq 1$ where $n$ is the LL number. Only in the LLs with $n \geq 1$ (i.e., for relatively weaker magnetic field in comparison to the LLL, $n=0$ ) are cyclotron orbits of sufficiently large size to match every second, third particle, and so on. This is because the size of the cyclotron orbits in $n$th LL grows proportionally to the factor $2 n+1$ present in Landau kinetical energy in higher LLs, that is, the cyclotron orbit size attains the value $A=\frac{(2 n+1) h c}{e B}$ for particles with kinetic energy $(2 n+1) \frac{e B}{2 m c}$ in $n$th LL. Moreover, for $n \geq 1$ too short cyclotron orbits may occur as well as in the LLL, but in higher LLs rather close the subbands edges, that is, for sufficiently small density of particles and thus larger separation which can commensurate with longer cyclotron orbits at $n \geq 1$. Let us emphasize, however, that the quantization of the transverse resistance $R_{x y}$ related to any fractional-filling rates $v$ (also in higher LLs) is always as for ordinary FQHE prerequisite from the LLL, that is, equal to $\frac{h}{e^{2} y}$, but the correlations expressed by the exponent in the Jastrow polynomial vary displaying singlelooped or multilooped braid exchanges, both accessible in higher LLs in the sharp opposition to the LLL (where only multilooped orbits were possible for fractional fillings $v<1$ ).

\subsection{Hierarchy for $F Q H E$ in graphene}

Due to the specific band structure in graphene with Dirac points on the corners of the hexagonal Brillouin zone [19], the LL spectrum is not equidistant as for ordinary 2DEG but is proportional to $\sqrt{n}$ instead of $n$ ( $n$ is the number of the LL) [28]. This specific "relativistic" form of LL energy emerges from the linearity in the momentum of the local Hamiltonian to the vicinity of Dirac points - this Hamiltonian determines carrier quantization in graphene. Note, however, that the degeneracy of each LL subband in graphene is the same one as in the conventional 2DEG and equals $\frac{B S}{h c / e}$. Nevertheless, the number of subbands per each LL in graphene is different from the conventional semiconductor case and equals 4 in graphene, 
which corresponds to the Zeeman spin splitting and to the valley splitting (absent in conventional semiconductors) due to two inequivalent Dirac points mixed with two sublattices in crystal lattice of the graphene sheet [28]. The Zeeman splitting in graphene is small [19], and the valley splitting is small as well [28], thus the fourfold approximate spin-valley additional degeneracy may be assumed. It results in the following form of IQHE hierarchy in monolayer graphene $v=4\left(n+\frac{1}{2}\right)$ in agreement with experimental observations [19]. The remarkable difference with respect to the ordinary 2DEG resoles itself to the factor 2 instead of 4, due to only spin degeneracy in conventional 2DEG, and the presence of the shift by 2 . This shift is due to the Berry phase in monolayer graphene and sharing of the LLL states between particles and holes from the conduction and valence band at the zero-energy level [28]. Hence, the bottom of the LLL is shifted by 2 (in terms of the filling factor) upward. Conventionally, filling rates for holes from the valence band are assigned as negative numbers mirror reflection of those positive numbers denoting filling rates for electrons from the conduction band. An additional opportunity in graphene, beyond the ability of conventional 2DEG, is the possibility to control a transition between particles and holes in graphene by the shift of the Fermi level passing the Dirac point. Experimentally, it is realized by the application of a lateral relatively small voltage. The above-mentioned Berry phase contribution to the Landau energy spectrum in graphene manifests itself by an additional $\pi$ phase shift born by the chiral valley pseudospin [28].

For the bilayer graphene, the situation slightly differs [28, 29]. Due to an interlayer hopping, the Hamiltonian for bilayer graphene attains back the quadratic form with respect to the momentum. Hence, the LL spectrum in bilayer graphene resembles that one for the ordinary 2DEG but with four subbands for each LL level except for the LLL, which has eightfold degeneration $[28,29]$. As usual in graphene, the division of the LLL subbands equally among particles and holes causes the bottom for uniformly charged carriers (electrons or holes) to be placed in the center of the eightfold-degenerated LLL (let us remind that in the monolayer graphene, the LLL was only fourfold degenerated; the additional degeneracy in the bilayer graphene is caused by the inclusion of states with $n=0$ and $n=1$ to the LLL in the bilayer graphene in opposition to the monolayer one). In the bilayer graphene, the Berry phase shift for chiral particles is also different in comparison to the monolayer one and equals $2 \pi$ [28]. Hence, the consecutive plateaus of IQHE are located in bilayer graphene at integer-filling rates, whereas in monolayer graphene, they were located at half-fillings of the LLs [28, 29].

\subsection{FQHE hierarchy in monolayer graphene}

For the magnetic field strong enough that $v \in(0,1)$ and for Fermi level shifted (by a lateral voltage) to the conduction band, we deal with fractionally filled first conduction subband of the particle LLL $n=0,2 \uparrow$ (in this numeration of subbands 2 marks the valley pseudospin orientation and the arrow $\uparrow$ marks the orientation of the ordinary spin). For $N<N_{0}$ the filling rate, $v=N / N_{0}$, is fractional (the degeneracy $N_{0}$ of each subband is $N_{0}=\frac{B S}{h c / e}$ )

An important property follows from the fact that cyclotron orbits in graphene must be accommodated to the bare kinetic energy $T=\hbar \omega_{c}\left(n+\frac{1}{2}\right)$ with $\omega_{c}=\frac{e B}{m^{\prime}}$, similarly in the conventional semiconductor 2DEG (as in non-interacting 2D gas), despite the different "relativistic" version of Landau levels energy in graphene. This "relativistic" oddness is caused, however, by peculiar 
crystal field features in graphene which do not change the topology of braids. Thus, the bare kinetic part of Landau energy is not modified by the crystal field and the dimension of braid cyclotron orbits repeat the corresponding orbit size from the non-interacting gas. Therefore, for graphene the cyclotron orbit structure is governed by ordinary Landau level restrictions despite a specific band structure with Dirac points, being the result of the crystal field in graphene and not of kinetic energy origin. The difference between the conventional 2DEG system and graphene will be thus related with the different number of LL subbands in graphene in comparison to the conventional 2DEG. The uniform shift in filling factors will be caused also by the Barry phase shift, nonzero for chiral valley pseudospin in graphene [28].

Therefore, the cyclotron orbit size in the subband $n=0,2 \uparrow$ is equal to $\frac{h c / e}{B}=\frac{S}{N_{0}}$ ( $S$ is the sample surface). Because this orbit size is lower than the interparticle spacing expressed by $\frac{S}{N}\left(\right.$ as $\left.N<N_{0}\right)$, the multilooped braids are needed to match neighboring particles. The commensurability condition reads here as $q \frac{S}{N_{0}}=\frac{S}{N^{\prime}}$, which gives $v=\frac{N}{N_{0}}=\frac{1}{q^{\prime}}(q-$ odd integer [21]). For the local band holes in this subband, the particle-hole symmetric-filling rates $v=1-\frac{1}{q}$ are expected.

One can generalize this fundamental fractional series by an assumption that in the case when the portion of the residual flux per last loop must be different than the portions per former loops, the last loop of the multilooped cyclotron orbit may be commensurate with every $l$ th particle separation, whereas the former loops take away an integer number of flux quanta (are commensurate with neighboring particles). For $l=2,3, \ldots$, the last loop reaches every $l$ th particles (next neighbors). In this manner, we obtain the hierarchy of fillings for FQHE in this LLL subband in the following form: $v=\frac{l}{l(q-1) \pm 1}, v=1-\frac{l}{l(q-1) \pm 1}$, where $l=1,2, \ldots$ and minus in the denominator corresponds to the possibility of the reverse eight-figure orientation of the last loop with respect to the antecedent loops in the multilooped orbit. Additionally, we notice that the Hall metal states can be achieved in the limit $l \rightarrow \infty$ in the above formula, which corresponds to the situation when the residual flux passing through the last loop tends to zero. This means that the last loop can reach in such a case the infinitely distant particles as for fermions at the absence of the magnetic field, which is referred to the case of the Hall metal archetype for $v=\frac{1}{2}$ in the conventional 2DEG. In the limit right arrow $\infty$, we arrive thus with the hierarchy for the Hall metal states in the form: $v=\frac{1}{q-1}, v=1-\frac{1}{q-1}$.

One can observe also that other variants of commensurability may be concerned with multilooped orbits. Namely, each loop of the multilooped structure may be in principle accommodated to particle separation in a different and mutually independent manner matching nearest and next neighbors in various schemes. One of such a possibility may correspond with the situation when in $q$-looped orbit $q-1$ loops are accommodated to every $x$ th particle $(x=1,2,3, \ldots)$, whereas the last one fits to every $l$ th particle separation. The similar commensurability scheme is observed in ordinary 2DEG Hall systems within the LLL for exotic fractions, for example, $v=\frac{4}{11}, \frac{5}{13}, \frac{3}{8}, \frac{3}{10}$ (beyond the CF hierarchy). It is noticeable, however, that a similar series of exotic FQHE-filling fractions are not observed in the LLL in graphene up to date, though it is observed in the first LL in monolayer graphene and also in bilayer graphene (as will be analyzed below). 
In order to include the Berry phase anomaly in graphene, the overall shift of $v$ by -2 must be performed (for monolayer case), but we confine our notation to the net-filling fractions, neglecting this uniform shift.

With lowering of the magnetic field, one can achieve the completely filled subband $n=0,2 \uparrow$, which corresponds to $v=1$ and the IQHE state. For still lower magnetic fields, the last subband of LLL $n=0,2 \downarrow$ is gradually filled by electrons. In this subband, the cyclotron orbit size $\frac{S}{N_{0}}$ is still lower than the interparticle separation $\frac{S}{N-N_{0}}$ (because $N-N_{0}<N_{0}$ ) similarly in the antecedent subband. Therefore, the multilooped structure of orbits must be repeated here from the previous subband. The FQHE hierarchy is exactly the same as for antecedent subband, only shifted ahead by 1 . After complete filling of this subband, the LLL is completely filled, that is, there are all four subbands of the LLL filled. This gives the IQHE according to its main-line hierarchy $v=4\left(n+\frac{1}{2}\right)$.

In an analogous way, one can consider fillings of the following LLs. The nearest one corresponds to $n=1$. This level has four subbands of electron type. Let us emphasize, however, that the bare kinetic energy in this level (in all its subbands) is equal to $\frac{3 \hbar \omega_{c}}{2}$ and the related cyclotron orbit size is $\frac{3 h c}{e B}=\frac{3 S}{N_{0}}$ (larger in comparison to the LLL). When $N \in\left(2 N_{0}, 3 N_{0}\right]$, then electrons fill the $n=1,1 \uparrow$ subband. In this subband, the cyclotron orbits are of size $\frac{3 S}{N_{0}}$ and it must be accommodated with interparticle separation between electrons in this subband, $\frac{S}{N-2 N_{0}}$. For small amount of electrons in the subband, we thus can meet with the multilooped orbits if single-looped orbits are too short, $\frac{3 S}{N_{0}}<\frac{S}{N-2 N_{0}}$. The $q$-looped orbits satisfy the commensurability condition $q \frac{3 S}{N_{0}}=\frac{S}{N-2 N_{0}}, q$ - odd integer, which defines the main series for FQHE (multiloop) in this subband, $v=2+\frac{1}{3 q}$. This main line can be supplemented to the complete related hierarchy, $v=2+\frac{l}{l 3(q-1) \pm 1}, v=3-\frac{l}{l 3(q-1) \pm 1} l=i / 3, i=1,2, \ldots$, with the Hall metal hierarchy in the limit $l \rightarrow \infty$, as described in the case of the LLL. These series of FQHE filling rates are located closer to the subband edges in comparison to the FQHE rates in the LLL. Simultaneously, in the central part of this higher LL subband the new type of commensurability is possible, not accessible in the LLL. This new commensurability occurs when $\frac{3 S}{N_{0}}=\frac{x S}{N-2 N_{0}}$ and $x=1,2,3$ corresponding to fitting of the single-looped orbits (large enough in this subband) with every $x$ th particle ( $x$ order next-nearest neighbors). From this new commensurability opportunity, one finds fractions $v=\frac{7}{3}, \frac{8}{3}, 3$ corresponding to single-looped cyclotron orbits similar as for IQHE. Thus, for $v=\frac{7}{3}, \frac{8}{3}$ we deal with the FQHE (single loop). This is a new Hall feature manifesting itself only in higher LLs, where cyclotron orbits may be larger than the interparticle separation and orbits can reach next-nearest neighbors.

Finally, let us note that at the special case of the commensurability, $\frac{3 S}{N_{0}}=\frac{1.5 S}{N-2 N_{0^{\prime}}}$, one can meet at $v=\frac{5}{2}$. This commensurability concerns rather the paired particles and not the single ones. The pairing does not change the cyclotron radius but reduces twice the carrier number $\frac{N-2 N_{0}}{2}$, which gives the above commensurability for pairs at $v=\frac{5}{2}$. Hence, at this filling rate, one can expect a manifestation of the IQHE but for paired electrons (the considered correlation corresponds to $p$-like pairing due to the spin polarization in this subband).

An interesting new possibility for commensurability happens next for $q$-looped orbits. As mentioned previously, the size of particular loops in the multilooped structure may be in general accommodated to the interparticle spacing in an independent manner resulting in the 
abundance of possible new filling rates. The loop size may be accommodated to $x$ th-order next-nearest neighbors independently. In particular, it results in the additional hierarchy in all subbands of the first LL, $\quad v=2(3,4,5)+\frac{x l}{l 3(q-1) \pm 1}, \quad v=3(4,5,6)-\frac{x l}{b 3(q-1) \pm 1}, \quad$ which for $q=3, x=2,3, l=i / 3, i=1,2,3 \quad$ reproduces $\quad v=\frac{7}{3}, \frac{8}{3}, \frac{12}{5}, \frac{13}{5}, \frac{17}{7}, \frac{18}{7}, \frac{22}{9}, \frac{23}{9}, \frac{10}{3}, \frac{11}{3}, \frac{17}{5}, \frac{18}{5}, \frac{24}{7}, \frac{25}{7}, \frac{13}{3}, \frac{14}{3}, \frac{22}{5}, \frac{23}{5}$. This opportunity for FQHE pretty well agrees with the recent observations of FQHE in three first subbands of the $n=1 \mathrm{LL}$ in monolayer graphene at ultra-low temperatures [16].

The similar scheme of commensurability may be applied to the following subbands. For the subband $n=1,1 \downarrow$, the cyclotron orbit size is again equal to $\frac{3 h c}{e B}=\frac{3 S}{N_{0^{\prime}}}$, whereas the interparticle spacing is governed by plaque $\frac{S}{N-3 N_{N^{\prime}}}$, for $N \in\left(3 N_{0}, 4 N_{0}\right)$. The basic commensurability condition has thus the form: $\frac{q 3 S}{N_{0}}=\frac{S}{N-3 N_{0}}$ which gives the main series for FQHE (multiloop), $v=3+\frac{1}{3 q^{*}}$. This series can be developed to the full hierarchy via more complicated commensurability opportunities, as described above. In this way, the condition $\frac{3 S}{N_{0}}=\frac{x S}{N-3 N_{0}}$ with $x=1,2,3$ gives fractions with single-looped correlations of FQHE (single-loop)-type for $v=\frac{10}{3}, \frac{11}{3}$ and fully developed IQHE for $v=4$. A possible paired state can be realized in this subband at $v=\frac{7}{2}$.

\subsection{FQHE hierarchy in bilayer graphene}

In bilayer graphene, the topology of braid trajectories considerably changes in comparison to the monolayer system. The bilayer graphene is not strictly two-dimensional and the bilayer graphene opens new possibility for the topology of trajectories. Two sheets of the graphene plane lie in close distance and electrons can hop between these two sheets. We deal here with electrons residing on a two-sheet structure instead of the single sheet as it was the case for the monolayer graphene.

All the above-described requirements to fulfill commensurability condition in order to define the related braid group are binding also for the bilayer graphene, with a single exception. This exception is linked with the fact that double-looped cyclotron orbits may have in bilayer graphene the same size as the single-looped orbit, that is, the one additional loop may not enhance the cyclotron radius. This is in opposition to the monolayer case, where the additional loops always enhance cyclotron radius. This difference in bilayer graphene follows, however, from the fact that the second loop may be located in the opposite sheet of bilayer graphene with respect to the first one and the external field passing through such a double-looped orbit is twice larger than the flux passing through the single-looped orbit (and the double-looped orbit ideally planar as well). When the two loops are located in the distinct sheet of the bilayer structure, then each loop has its own and separate surface, as usual in 3D (here reduced, however, to double-sheet structure). This is in sharp distinction from the multilooped (double-looped, in particular) cyclotron orbit located in the purely 2D plane (like monolayer graphene). More generally, in the bilayer system loops of the multilooped orbit may be located partly in both 2D sheets. To account this effect in topological terms adjusted to braid trajectories and commensurability requirements, one must neglect the contribution of the one loop in the multilooped structure when the total flux of the external field is divided for fractions per all loops. This one loop may capture its own additional flux, whereas the remaining loops must share the same flux similarly as passing through any cyclotron orbit in monolayer case. 
Removing the single loop must be done independently from how the loops are distributed among two sheets. When we consider a selected loop located in the opposite sheet with respect to the antecedent loop, the next loops must fill both sheets of bilayer structure as additional ones regardless their specific distribution-thus these all loops take part in dividing the external field flux exactly in the same manner as in monolayer case provided that the selected loop is omitted together with the flux passing this loop. This trick reduces the bilayer system to monolayer one in braid-loop topology sense. Thus, we can denote the commensurability condition in the bilayer graphene for the usual case of too short single-looped cyclotron orbits in the following form (as an example, for the subband $n=0,2 \uparrow$ of the LLL-i.e., the first particle-type subband of the LLL),

$$
\begin{aligned}
& (p-1) \frac{h c}{e B}=\frac{S}{N}, \\
& v=\frac{N}{N_{0}}=\frac{1}{p-1}=\frac{1}{2}, \frac{1}{4}, \frac{1}{6}, \ldots,
\end{aligned}
$$

here $N$ is the total number of particles counted in both graphene sheets, $N_{0}=\frac{B S e}{h c}$ is the degeneracy also for both sheets together, $S$ is the surface of the sample (the surface of the single sheet), $p$ is an odd integer (it must be odd in order to assure that half of the cyclotron orbit defines the braid, similarly as in monolayer case).

The factor $p-1$ in 1.h.s. of Eq. (1) is caused by the fact that in an enhancement of the effective cyclotron orbit size participates only orbits from the ideally 2D sheet of bilayer graphene (no matter where the doubling loops are located) with exception of a single loop which may be located in opposite sheet to the former loop, and thus must be avoided in order to reduce the bilayer system to an effective monolayer one.

The contribution of this sole loop is independent from other loops and must be omitted. After avoidance of this single loop, the next loops must duplicate the former ones without any rise of the surface per total cyclotron orbit no matter in which way loops are distributed among both sheets (in both sheets, the remaining loops will duplicate loops already present there). Thus, only $p-1$ loops take part in the enhancement of the effective $p$-looped cyclotron orbit in bilayer graphene.

It must be emphasized that for multilooped orbits in bilayer graphene the total number of loops still is $p$ (despite avoiding one loop at the formulation of the commensurability condition) - therefore, the generators of the corresponding cyclotron subgroup are of the form $\sigma_{j}^{p}$, which results in the standard Laughlin correlations with the Jastrow polynomial with $p$ exponent. However, due to the specific for bilayer structure commensurability (1) the resulting main line of filling fractions is $v=\frac{1}{p-1}$ ( $p$-odd) in the first particle-type subband of the LLL, that is, in the subband $n=0,2 \uparrow$. The even denominator in this main series for the FQHE hierarchy for bilayer graphene pretty well coincides with the experimental observations [10].

For holes in this subband (holes corresponding to empty states in the almost filled subband of particle type), we can write, $v=1-\frac{1}{p-1}$. The generalization to the full hierarchy of FQHE in this subband attains thus the form, $v=\frac{l}{l(p-2) \pm 1}, v=1-\frac{l}{l(p-2) \pm 1}$, where $l>1$ corresponds to some filling factor for other correlated Hall state, similarly as discussed in the monolayer case. In 
the following subbands of the LLL, $n=0,2 \downarrow$ (assuming that this subband succeeds the former one), the hierarchy is repeated in the same form only uniformly shifted ahead by one (because the commensurability conditions are similar for all subbands with the same $n$ due to the same size of the cyclotron orbits).

The considerable novelty occurs, however, in the next two subbands of the LLL, $n=1,2 \uparrow$ and $n=1,2 \downarrow$. Because of the larger size of cyclotron orbits for $n=1$, the FQHE main series in the first of these subbands of the LLL, $n=1,2 \uparrow$, attains the form,

$$
\begin{aligned}
& \frac{3 h c}{e B}=\frac{3 S}{N_{0}}<\frac{S}{N-2 N_{0}}, \\
& (p-1) 3 \frac{h c}{e B}=(p-1) \frac{3 S}{N_{0}}=\frac{S}{N-2 N_{0}}, \\
& v=\frac{N}{N_{0}}=2+\frac{1}{3(p-1)}=2+\frac{1}{6}, 2+\frac{1}{12}, 2+\frac{1}{18}, \ldots,
\end{aligned}
$$

The generalization of this main series for holes in the subband and to the full FQHE hierarchy in this subband looks as follows: for subband holes, $v=3-\frac{1}{3(p-1)}$ and for the full FQHE hierarchy in this subband, $v=2+\frac{l}{\sqrt{3(p-2) \pm 1}}, v=3-\frac{l}{\sqrt{3(p-2) \pm 1}}, l=\frac{i}{3}, i=1,2,3, \ldots$ (the Hall metal hierarchy may be obtained in the limit $l \rightarrow \infty$ ).

In the subband $n=1,2 \uparrow$ of the LLL, the new commensurability opportunity occurs (a similar one as that which occurred in the first LL of the monolayer graphene): $\frac{3}{N_{0}}=\frac{x}{N-2 N_{0}}$ for $x=1,2,3$. It gives filling ratios $v=\frac{7}{3}, \frac{8}{3}, 3$. These rates are related with single-looped cyclotron trajectories, thus with single-loop correlations similar as for IQHE (though the first two for not integerfilling rates). This is typical for LLs with $n \geq 1$ Hall features we called as FQHE (single loop). Similarly as in the monolayer case one can consider paired state for $x=1.5$ in the above formula, which corresponds to the perfect commensurability of cyclotron orbits of electron pairs with the separation of these pairs at electron-filling rate $v=\frac{5}{2}$.

Filling of the last subband $n=1,2 \downarrow$ in the LLL in bilayer graphene undergoes the similar constraints as for all subbands with $n=1$, the cyclotron orbits have the same size. Thus, the FQHE hierarchy is only shifted by 1 from the antecedent subband. The situation changes, however, in the next LL (the first one beyond the LLL). The cyclotron orbits are determined here by the bare kinetic energy for $n=2$, which gives the orbit size: $\frac{5 h c}{e B}=\frac{5 S}{N_{0}}$. The similar analysis as in the LLL gives in the subband $n=2,1 \uparrow$ the main series and the full hierarchy for FQHE (multiloop) $v=4+\frac{1}{5(p-1)}, v=4+\frac{l}{\sqrt{5(p-2) \pm 1}}, l=\frac{i}{5}, i>1$, respectively (for subband holes, the substitution of $4+$ by $5-$ in both the above formulae is needed). As previously, the limit $l \rightarrow \infty$ determines the Hall metal hierarchy. The next feature in this subband is the presence of four (instead of two) satellite FQHE (single-loop) states symmetrically located around the central paired state. In the subband $n=2,1, \uparrow$, these satellite states occur at $v=\frac{21}{5}, \frac{22}{5}, \frac{23}{5}, \frac{24}{5}$ and the central paired state at $v=\frac{9}{2}$. This similar hierarchy scheme is repeated in all four subbands of the first LL with $n-2$.

The evolution of the fractional-filling hierarchy of subsequent LLs is pictorially presented in Figures 4 and $\mathbf{5}$ for the monolayer and bilayer graphene, correspondingly. It is also summarized in Tables 1 and 2. 

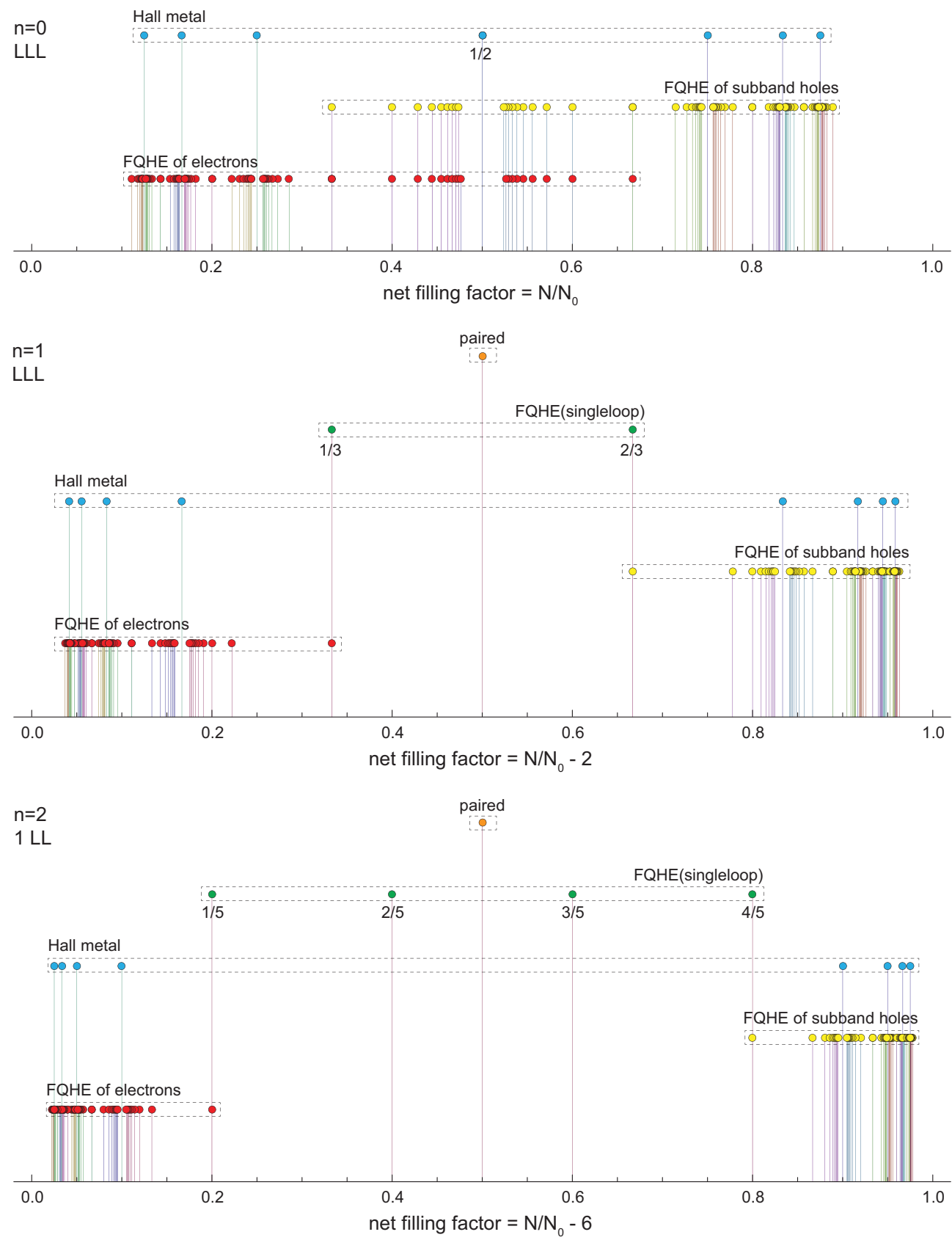

Figure 4. Illustration of the FQHE hierarchy in three first LLs of the monolayer graphene; for each LL the first particle subband is illustrated, the next subbands in each LL repeat the hierarchy from the first one. Different types of ordering are indicated with spikes of various heights. Series for ordinary FQHE (multiloop), FQHE (single-loop), Hall metal, and paired state are displayed acc. to the hierarchy described in Table 1 with $q=3-9,1=1-10$; only a few selected ratios from these series are explicitly written out. 

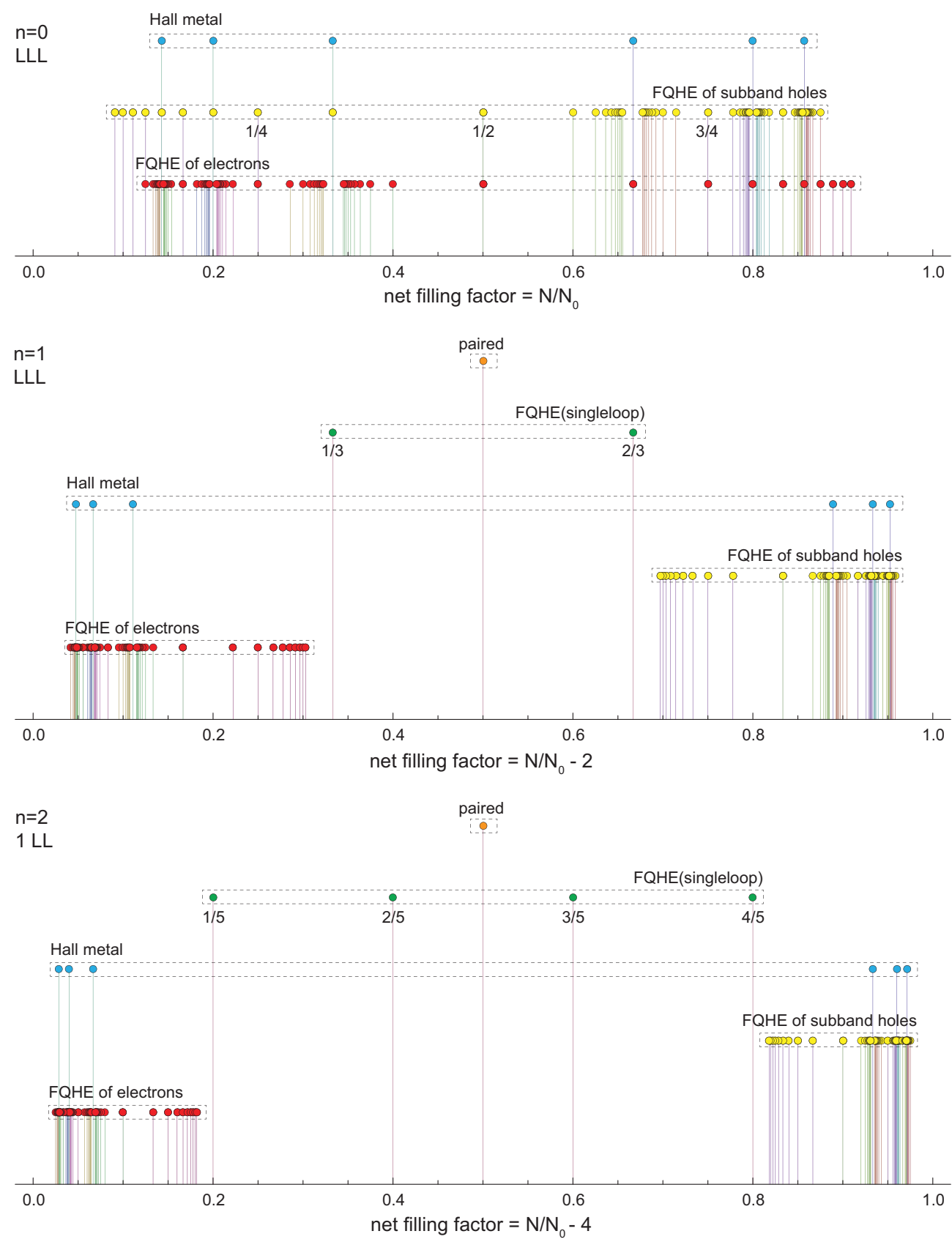

Figure 5. FQHE hierarchy in two first LLs of the bilayer graphene; for the LLL two subbands with $n=0$ and $n=1$ are included. Different types of braid correlations are indicated with spikes with various heights. Hierarchy series are displayed acc. to the formulae presented in Table 2 with $q=3-9, i=1-10$; limited number of ratios from these series are explicitly written out. 


\begin{tabular}{llll}
\hline LL subb. & FQHE (single-loop), paired, IQHE & $\begin{array}{l}\text { FQHE (multiloop) } \\
\left(q-\text { odd, } l=\frac{i}{2 n+1}, i=1,2,3, \ldots\right)\end{array}$ & Hall metal \\
\hline$n=0,2 \uparrow$ & 1 & $\frac{1}{q}, 1-\frac{1}{q}, \frac{l}{(q-1) \pm 1}, 1-\frac{1}{1(q-1) \pm 1}$ & $\frac{1}{q-1}, 1-\frac{1}{q-1}$ \\
$n=1,1 \uparrow$ & $\frac{7}{3}, \frac{8}{3},\left(\frac{5}{2}\right.$ paired), 2,3 & $2+\frac{1}{3 q}, 3-\frac{1}{3 q}, 2+\frac{l}{3(q-1) \pm 1}, 3-\frac{l}{3(q-1) \pm 1}$ & $2+\frac{1}{3(q-1)}, 3-\frac{1}{3(q-1)}$ \\
$n=2,1 \uparrow$ & $\frac{31}{5}, \frac{32}{5}, \frac{33}{5}, \frac{34}{5}\left(\frac{13}{2}\right.$ paired), 6,7 & $6+\frac{1}{5 q}, 7-\frac{1}{5 q}, 6+\frac{l}{5((q-1) \pm 1}, 7-\frac{1}{5(q-1) \pm 1}$ & $6+\frac{1}{5(q-1)}, 7-\frac{1}{5(q-1)}$ \\
\hline
\end{tabular}

Table 1. LL-filling factors for FQHE determined by commensurability condition (paired indicates condensate of electron pairs), for the first particle subband in each of the three first LLs $(n=0,1,2)$ for the monolayer graphene.

\begin{tabular}{llll}
\hline LL subb. & $\begin{array}{l}\text { FQHE (single-loop), paired, } \\
\text { IQHE }\end{array}$ & $\begin{array}{l}\text { FQHE (multiloop) }\left(q-\text { odd, } l=\frac{i}{2 n+1},\right. \\
i=1,2,3, \ldots)\end{array}$ & Hall metal \\
\hline $\begin{array}{l}n=0,2 \uparrow \\
\text { (LLL) }\end{array}$ & 1 & $\frac{1}{(q-1)}, 1-\frac{1}{(q-1)}, \frac{l}{((q-2) \pm 1}, 1-\frac{l}{l(q-2) \pm 1}$ & $\frac{1}{q-2}, 1-\frac{1}{q-2}$ \\
$n=1,2 \uparrow$ & $\frac{7}{3}, \frac{8}{3},\left(\frac{5}{2}\right.$ paired $), 2,3$ & $2+\frac{1}{3(q-1)}, 3-\frac{1}{3(q-1)}, 2+\frac{l}{3(q-2) \pm 1}, 3-\frac{l}{3(q-2) \pm 1}$ & $2+\frac{1}{3(q-2)}, 3-\frac{1}{3(q-2)}$ \\
(LLL) & & $4+\frac{1}{5(q-1)}, 4+\frac{l}{5((q-2) \pm 1}, 5-\frac{1}{5(q-1)}, 5-\frac{l}{5(q-2) \pm 1}$ & $4+\frac{1}{5(q-2)}, 5-\frac{1}{5(q-2)}$ \\
$n=2,1 \uparrow$ & $\frac{21}{5}, \frac{22}{5}, \frac{23}{5}, \frac{24}{5},\left(\frac{9}{2}\right.$ paired) $, 4,5$ &
\end{tabular}

Table 2. FQHE hierarchy found by commensurability braid group approach (paired marks condensate of electron pairs), in the first subbands of two first LLs ( $n=0,1$ correspond to the LLL whereas $n=2$ for the first LL beyond the LLL) in the bilayer graphene.

\subsection{Specific to bilayer graphene FQHE hierarchy change caused by the type of the LLL degeneracy lifting}

For bilayer graphene the degeneration of $n=0$ and $n=1$ states results in eightfold degeneracy of the LLL, doubling fourfold spin-valley degeneracy of the LLL in comparison to the monolayer case. The degeneracy is not exact and for enhancing magnetic field amplitude both the Zeeman splitting and the valley splitting grow. Stress, deformation, and structure imperfections also cause lifting of the valley degeneracy. Moreover, the Coulomb interaction causes mixing of $n=0,1$ states lifting their degeneracy within the LLL. Of particular importance is, however, an order of subbands after the degeneracy lifting allowing for mutually inverted ordering of LLL subbands with distinct $n=0,1$. The orders $n=0,1$ or $n=1,0$ lead to distinct FQHE-filling hierarchy. In the case when the LLL subbands with $n=$ 1 is filled earlier than the $n=0$ subband, one arrives with the following hierarchy for the first subband $n=1,2 \uparrow$ : multilooped orbits for $v=\frac{l}{13(p-2) \pm 1}, v=1-\frac{l}{13(p-2) \pm 1}$, single-looped orbits for $v=\frac{1}{3}, \frac{2}{3}$, and a paired state for $v=\frac{1}{2}$. For the next subband, $n=0,2 \uparrow$, we get the hierarchy in the form: multilooped orbits for $v=1+\frac{l}{\Gamma(p-2) \pm 1}, v=2-\frac{l}{l(p-2) \pm 1}$ and no singlelooped orbits. The comparison of reverted orderings of two first LLL subbands is summarized in Table 3.

One can consider also the situation in the LLL of bilayer graphene, when the degeneracy of $n=0,1$ states is lifted in such a way that both levels cross at certain filling factor $v^{*}<1$ (cf. Ref. [30], where mixing between $n=0,1$ states has been analyzed numerically on small models on torus or sphere). Let us assume for an example, that the $n=1$ subband 


\begin{tabular}{llll}
\hline LL subb. & FQHE (single-loop), paired, IQHE & FQHE (multiloop) $\left(q-\right.$ odd, $\left.l=\frac{i}{2 n+1}, i=1,2,3, \ldots\right)$ & Hall metal \\
\hline$n=0,2, \uparrow$ & 1 & $\frac{1}{(q-1)}, 1-\frac{1}{(q-1)}, \frac{l}{l(q-2) \pm 1}, 1-\frac{l}{l(q-2) \pm 1}$ & $\frac{1}{q-2}, 1-\frac{1}{q-2}$ \\
$n=1,2 \uparrow$ & $\frac{4}{3}, \frac{5}{3},\left(\frac{3}{2}\right.$ paired $), 1,2$ & $1+\frac{1}{3(q-1)}, 2-\frac{1}{3(q-1)}, 1+\frac{l}{3(q-2) \pm 1}, 2+\frac{l}{3(q-2) \pm 1}$ & $1+\frac{1}{3(q-2)}, 2-\frac{1}{3(q-2)}$ \\
$n=1,2 \uparrow$ & $\frac{1}{3}, \frac{2}{3},\left(\frac{1}{2}\right.$ paired $), 1$ & $\frac{1}{3(q-1)}, 1-\frac{1}{3(q-1)}, \frac{l}{3(q-2) \pm 1}, 1-\frac{l}{3(q-2) \pm 1}$ & $\frac{1}{3(q-2)}, 1-\frac{1}{3(q-2)}$ \\
$n=0,2 \uparrow$ & 1,2 & $1+\frac{1}{q-1}, 1+\frac{l}{1(q-1) \pm 1}, 2-\frac{1}{q-1}, 2-\frac{l}{\frac{l(q-2) \pm 1}{2}}$ & $1+\frac{1}{q-2}, 2-\frac{1}{q-2}$ \\
\hline
\end{tabular}

Table 3. Comparison of filling hierarchy in the LLL level in the bilayer graphene for two mutually inverted successions of two lowest subbands: $n=0,2 \uparrow n=1,2 \uparrow$ (upper) and $n=1,2 \uparrow n=0,2 \uparrow$ (lower).

( $n=1,2 \uparrow)$ is energetically favorable up to some filling fraction $v^{*}$. At this filling rate, the subband $n=1,2 \uparrow$ crosses with the subband $n=0,2 \uparrow$ and the latter starts to be more convenient for $1+v^{*}>v>v^{*}$. The hierarchy of fractional fillings corresponding to such a situation looks like for ordinary filling of the subband $n=1,2 \uparrow$, though with an insertion of $n=0,2 \uparrow$ subband hierarchy. Depending on the value of $v^{*}$, the various patterns are possible by a combination of hierarchy patterns as illustrated in Table 3.

\section{Comparison with experiment}

The filling factor in graphene (monolayer and bilayer) can be changed both by the external magnetic field strength variation and by the particle concentration variation via shifting of the Fermi level near the Dirac point by the application of a lateral voltage of relatively small magnitude (typically 10-60 V). Due to spin-valley degeneracy and Berry phase contribution related with the chiral valley pseudospin, the IQHE is observed in monolayer graphene for fillings $v=4\left(n+\frac{1}{2}\right)=2,6,10,14, \ldots$ for particles from the conduction band and for the mirrornegative fillings for holes from the valence band. However, FQHE was not detected in graphene samples deposited on a substrate of $\mathrm{SiO}_{2}$, despite using very strong magnetic fields (up to $45 \mathrm{~T}$ ) [20]. Instead of FQHE features in this configuration in so strong magnetic fields, the emergence of additional plateaus of IQHE has been observed for the fillings $v=0, \pm 1, \pm 4$. This additional IQHE manifestation is linked with spin-valley degeneracy lifting due to an increase of the mass of Dirac fermions at stronger magnetic fields [20]. After mastering the technology of the so-called suspended ultra-small graphene scrapings with extreme purity and high mobility of carriers above $200,000 \mathrm{~cm}^{2} \mathrm{~V}^{-1} \mathrm{~s}^{-1}$, it was possible to observe FQHE in graphene at net fillings $v=1 / 3$ and $-1 / 3$ (the latter for holes in valence band) [31, 32]. Both these papers report the observation of FQHE for not extremely strong magnetic fields: in the paper [31], at the field of $14 \mathrm{~T}$, for electron concentration of $10^{11} / \mathrm{cm}^{2}$ and in the paper [32] at the field of $2 \mathrm{~T}$, but for a concentration smaller by one order of magnitude $\left(10^{10} / \mathrm{cm}^{2}\right.$ and the mobility of $200,000 \mathrm{~cm}^{2} \mathrm{~V}^{-1} \mathrm{~s}^{-1}$ ).

FQHE in suspended graphene is observed at relatively high temperatures around $10 \mathrm{~K}$ [33], and even higher (up to $20 \mathrm{~K}$ ) [34], which seems to be explained by the relative strengthening of the electric interaction due to the absence, for suspended samples, of a dielectric substrate 
(with the dielectric constant in case of $\mathrm{SiO}_{2}, \sim 3.9$ ), and on the other hand, with very high cyclotron energy in graphene (i.e., large energy gap between incompressible states).

The nowadays experimental data of FQHE in graphene [31-35] are consistent with the predictions of the braid group commensurability approach. In the case of graphene, the specific band structure with pseudo-relativistic conical Dirac bands leads to equal division of the LLL between the valence band of holes and the conduction band of electrons, which itself via an anomalous "relativistic" IQHE [20, 36, 37]. Applying lateral gate voltage (within the range ca. 5-60 V $[10,15,31])$ allows for the control of the density of carriers at a constant magnetic field, which is exceptionally valuable from an experimental point of view. For relatively small densities of carriers, the cyclotron orbits will be too short for braid exchanges of particles (leading to FQHE (multilooped)) at a relatively low magnetic field - the weaker for smaller concentrations is). The experimental observations exactly support this prediction [31, 32]. For low concentration, while closing on the Dirac point, one may expect, on the other hand, too strong which would correspond to an emergence of the insulating state near the Dirac point in a sufficiently large magnetic field. This prediction also agrees with the experiment [38]. In the case of the hexagonal structure of graphene, electron (or hole) Wigner crystallization [39] may exhibit interference between the triangular crystal sublattices, and including such resonance between two sublattices may cause blurring of the sharp transition to the insulator state, which is also consistent with observations.

The rapid progress in the recent experiment allowed for the precise observation of FQHE in graphene on the crystal substrate of boron nitride $(B N)$ in large magnetic fields of the order of $40 \mathrm{~T}$ (remarkably, FQHE features were noticed in this case up to $v=4$ ) $[15,16]$. This achievement is caused by avoiding crystalline disorder on the frontier of graphene layer with the substrate. For BN with similar graphene hexagonal surface lattice, the disorder perturbations are minimal which guarantee high mobility of carriers in graphene triggering FQHE organization.

The mobility of carriers in graphene is lower than in traditional 2DEG, but taking into account that the carrier concentration in graphene can be lower in comparison to semiconductor heterostructure [40,41], the corresponding mean free path in both cases well exceeds the sample dimension (of $\mu \mathrm{m}$ order, as the mobility is proportional to the concentration and to the mean free path of carriers).

It is worth noting that energy gaps protecting incompressible FQHE states in graphene are larger than in traditional semiconductor materials, reaching the high magnitude of the order of $10 \mathrm{~K}$, which is linked with Dirac massless character of carriers. In conventional semiconductor heterostructure, the corresponding gaps are much lower and the observed FQHE is much more fragile in comparison to graphene.

The recent development in experiments with monolayer graphene on BN substrate $[15,16]$ and with suspended small sheets [17, 18] allowed for the observation of more and more Hall features referred to FQHE in subsequent subbands of two first LLs. While the sequence of fillings in the lowest subband of the LLL fits well to CF predictions (including CFs with twoand four-flux quanta attached), an explanation of the FQHE-filling structure in next subbands strongly deviates from this simple picture. CF theory fails also in all subbands of the first LL 
[16-18]. Various scenarios of breaking of the approximate SU(4) spin-valley symmetry in graphene do not solve this problematic situation despite many theoretical attempts, which evidences insufficiency of the CF model in this case.

For much more effective understanding of FQHE in graphene, there occurs the braid group base commensurability approach. The hierarchy for FQHE predicted in this way is consistent with all experimental data known as of yet-the corresponding filling fractions can be reproduced by the topology braid group method (cf. Table 1). From this comparison, it is also clearly visible why the CFs are efficient only in the LLL and fails in many other situations.

Partial efficiency of CF model in the LLL is linked with the fact that exclusively in the LLL cyclotron orbits are always shorter than the interparticle spacing and additional loops are necessary. These loops can be simulated by fictitious field flux quanta attached to CFs. In the case, however, when the more complicated commensurability condition supports particular FQHE states in the LLL or in higher LLs the CF model fails. The braid group approach reproduces all features described correctly by CF model and moreover explains details inaccessible for CF approach. The usefulness of the CF model is in particular strongly limited in higher LLs because in this level the simple multilooped commensurability is needed only close to the subband edges, whereas the central regions of all subbands in higher LL are of single-looped type commensurability with next-nearest neighbors beyond the CF concept. For example, in the first LL on monolayer graphene the doublets of fillings are as follows: $\left(\frac{7}{3}, \frac{8}{3}\right),\left(\frac{10}{3}, \frac{11}{3}\right),\left(\frac{13}{3}, \frac{14}{3}\right),\left(\frac{16}{3}, \frac{17}{3}\right)$, corresponding to single-looped commensurability condition, not related to CFs. These doublets are visible in experiment [15-18]. The number of centrally located filling rates for FQHE (single loop) grows next with the LL number as $2 n$. The repeating doublet of filling ratios for $n=1$ is noticeable in very accurate measurements in suspended samples $[17,18]$ besides those on the BN substrate $[15,16]$. The observation [16] that the stability of FQHE(single-loop) states is of similar order as of IQHE states and higher in comparison to FQHE (multiloop) state features as is visible in Figure 6 is worth noting. This might be associated with stronger correlations related to the single-looped braids as for IQHE states.

The commensurability braid group approach successfully reproduces all the positions of observed features in two lowest LLs of the monolayer graphene. The elongate plateaus at edges of subbands with IQHE in center seem to embrace also minimums related to FQHE(multiloop) rates which are located closely to edges - such location makes them out of the experimental resolution. In higher LL, the new features were observed in between the abovementioned doublets but with not developed vanishing longitudinal resistivity as at other FQHE states. It suggests that not all particles participate in correlated state, but might correspond to multilooped correlation commensurate to every second or every third particles. And indeed, these new features in the first LL of monolayer graphene recently reported [16] at $v=\frac{7}{3}, \frac{8}{3}, \frac{12}{5}, \frac{13}{5}, \frac{17}{7}, \frac{18}{7}, \frac{22}{9}, \frac{23}{9}$, $\frac{10}{3}, \frac{11}{3}, \frac{17}{5}, \frac{18}{5}, \frac{24}{7}, \frac{25}{7}, \frac{13}{3}, \frac{14}{3}, \frac{22}{5}, \frac{23}{5}$ are reproduced one to one by the commensurability series $v=2(3,4)+$ $\frac{x l}{13(q-1) \pm 1}$ with $q=3, x=2,3, l=\frac{i}{3}, i=1,2,3$ as shown in Figure 6. One can notice that the FQHEfilling rates $\frac{7}{3}, \frac{8}{3}, \frac{10}{3}, \frac{11}{3}, \frac{13}{3}, \frac{14}{3}$ are repeated in this hierarchy. It is likely that the single-looped correlated states at the same filling rates are more stable than the multilooped ones which seem to be consistent with the experimental data presented in Figure 6 and in the upper panel of Figure 7. 


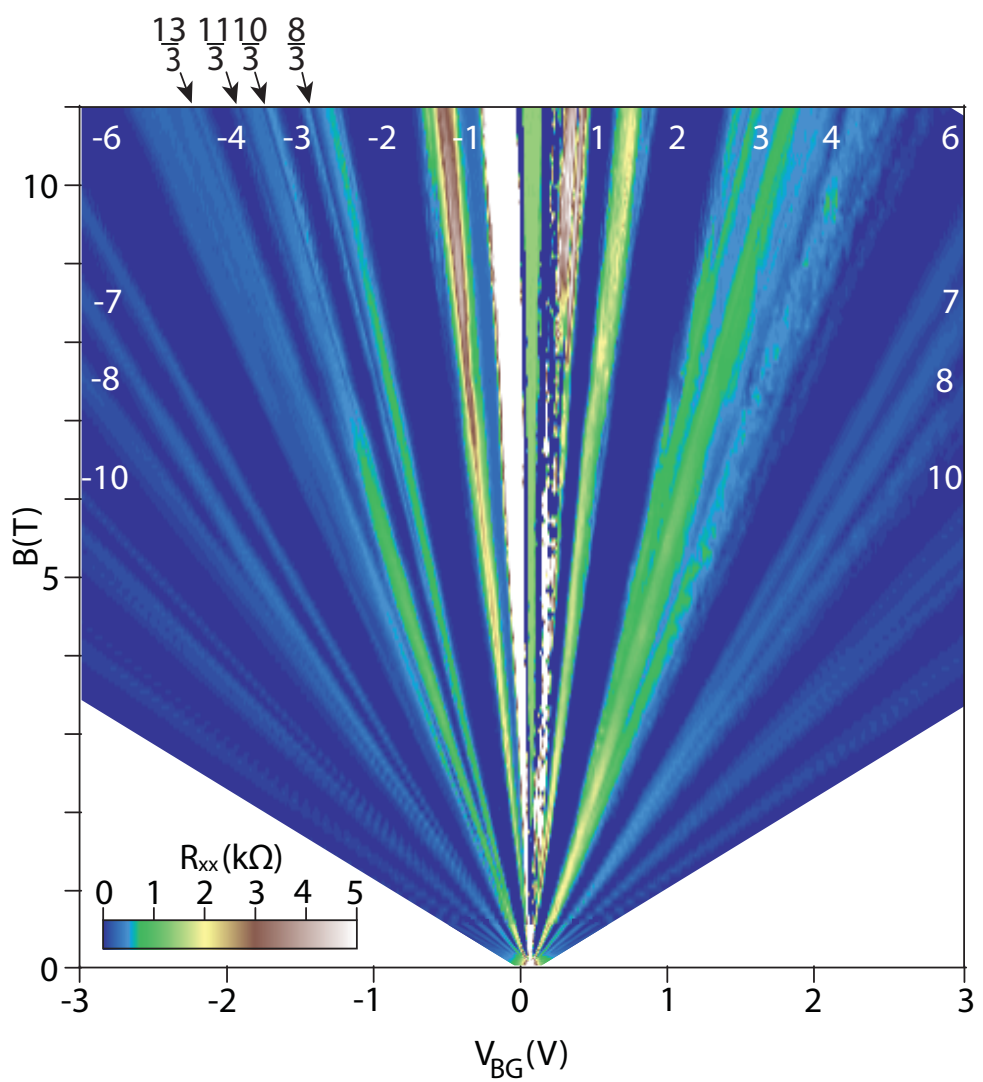

Figure 6. Fan diagram for $\rho_{x x}(v, B)$ for graphene up to $11 \mathrm{~T}$ (after [16]).

The next evidence supporting the correctness and usefulness of the commensurability condition is the consistence of the related predictions with the experimental observations in bilayer graphene. The remarkable feature observed in bilayer graphene is the occurrence of FQHE in the LLL in bilayer graphene at fractions with even denominators [10] which is completely beyond the CF model explanation ability. The commensurability braid group approach for bilayer graphene reproduces, however, perfectly all the experimentally observed FQHE hierarchy, including the controversial state at $v=-\frac{1}{2}-$ cf. Figure 8, the illustration in Figure 5, and in Table 2.

Note finally that the FQHE hierarchy in bilayer systems with the characteristic even denominators manifested itself previously also in bilayer 2DEG of conventional Hall setups-the existence of FQHE state at $v=\frac{1}{2}$ state has been discovered there $[42,43]$, which also is consistent with the commensurability braid group predictions.

The interesting observation in bilayer graphene is reported by the comparison of FQHE measurement in bilayer graphene in suspended samples and in samples on the BN substrate 

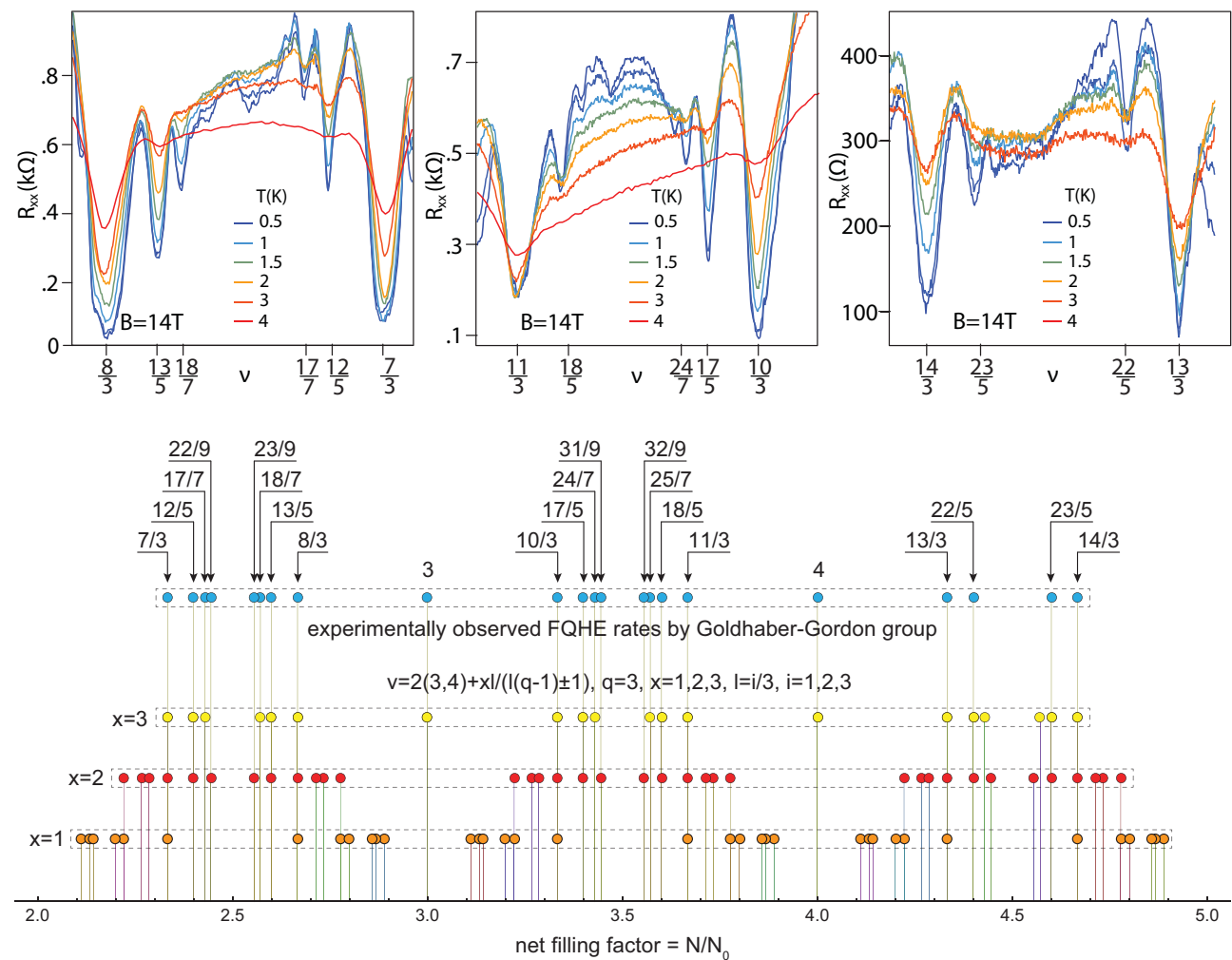

Figure 7. Not fully developed FQHE states with residual longitudinal resistance as corresponding to correlation of every second or every third particles at fractional rates reproduced by the commensurability series $v=2(3,4)+\frac{x l}{3(q-1) \pm 1}$ with $q=3, x=2,3, l=\frac{i}{3}, i=1,2,3$ (upper panels visualize longitudinal resistivity measurements after [16]).

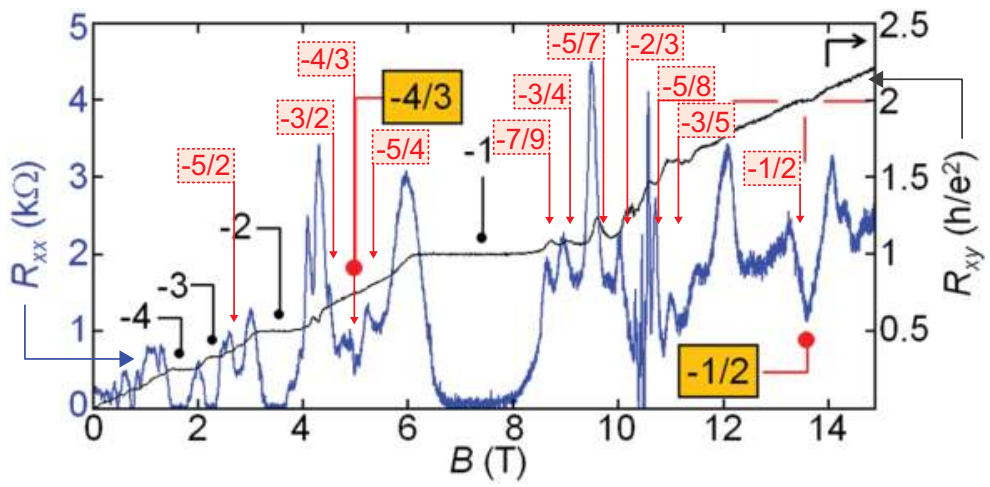

Figure 8. Observation of FQHE at $T=0.25 \mathrm{~K}$ in bilayer suspended graphene. (a) magneto-resistance $R_{x x}$ and $R_{x y}$ at the lateral voltage $-27 \mathrm{~V}$ (after [10]). In dotted frames, fitting with the hierarchy given in Table 2 is added. 
[10-14]. Surprisingly, the FQHE state at $v=-\frac{1}{2}$ observed in bilayer structure on BN substrate disappears in the suspended sample of bilayer graphene. We propose to explain this behavior by our commensurability braid group approach, noting that the occurrence of the FQHE state depends of the order of the LLL subband degeneracy lifting, as shown in Table 3. We suppose thus that the external conditions related to the presence of the BN substrate can reverse the ordering of the LLL subbands resulting in the occurrence of the FQHE state at $v=-\frac{1}{2}$, which is, however, no case for the suspended sample with opposite lowest subband ordering.

\section{Conclusion}

The commensurability of cyclotron orbits building cyclotron braids with interparticle spacing in homogeneous 2D-charged systems in magnetic field is formulated in order to verify the possibility of arrangement of correlated multiparticle Hall states and decipher the "magic" hierarchy of filling ratios for FQHE. By application of the commensurability braid group approach to study the statistics of interacting many particle systems, the FQHE hierarchy of filling rates in graphene has been determined. The evolution of this hierarchy with growing number of the LL has been deciphered in consistence with experimental observations. The new opportunities for commensurability in higher LLs were established leading to different than ordinary FQHE (multiloop)correlated states and the related fractional fillings of LLs have been identified beginning from the first LL. This new states have been named as FQHE (single loop) because the related correlations are described by single-looped braids. Both the monolayer and bilayer graphene have been analyzed and the essential difference of related hierarchy structures has been identified and described in detail. The even denominator main line of the fractional-filling hierarchy in the bilayer graphene is found in satisfactory consistence with the experimental observations. The presented hierarchy for the monolayer and bilayer graphene found the confirmation in available experimental data for graphene on BN substrate as well as for suspended samples including bilayer graphene for fillings up to sixth spin-valley subband. The impressive success of the commensurability braid group approach is the explanation of the disappearance of the FQHE at $v=-\frac{1}{2}$ in bilayer graphene in suspended sample, whereas this state has been formerly observed in bilayer graphene on the $\mathrm{BN}$ substrate. We link this phenomenon with the inversion of the ordering of the LLL lowest subbands caused by an influence of the crystalline BN substrate.

It is worth noting that the hierarchy observed in bilayer graphene is out of reach of the conventional CF theory.

\section{Acknowledgements}

This work is supported by the NCN projects P.2011/02/A/ST3/00116 and P.2016/21/D/ST3/ 00958. 


\section{Author details}

Janusz Edward Jacak

Address all correspondence to: janusz.jacak@pwr.edu.pl

Department of Quantum Technologies, Faculty of Fundamental Problems of Technology, Wroclaw University of Science and Technology, Wroclaw, Poland

\section{References}

[1] Tsui DC, Störmer HL, Gossard AC. Two-dimensional magnetotransport in the extreme quantum limit. Phys Rev Lett. 1982;48:1559.

[2] Laughlin RB. Anomalous quantum Hall effect: an incompressible quantum fluid with fractionally charged excitations. Phys Rev Lett. 1983;50:1395.

[3] Abrikosov AA, Gorkov LP, Dzialoshinskii IE. Methods of Quantum Field Theory in Statistical Physics. Dover: Dover Publ. Inc.; 1975.

[4] Jain JK. Composite Fermions. Cambridge: Cambridge UP; 2007.

[5] Jacak J, Gonczarek R, Jacak L, Jóźwiak I. Application of Braid Groups in 2D Hall System Physics: Composite Fermion Structure. Singapore: WorldScientific; 2012.

[6] Wu YS. General theory for quantum statistics in two dimensions. Phys Rev Lett. 1984;52:2103.

[7] Birman JS. Braids, Links and Mapping Class Groups. Princeton: Princeton UP; 1974.

[8] Imbo TD, Imbo CS, Sudarshan CS. Identical particles, exotic statistics and braid groups. Phys Lett B. 1990;234:103.

[9] Laidlaw MG, DeWitt CM. Feynman functional integrals for systems of indistinguishable particles. Phys Rev D. 1971;3:1375.

[10] Ki DK, Falko VI, Abanin DA, Morpurgo A. Observation of even denominator fractional quantum Hall effect in suspended bilayer graphene. Nano Lett. 2014;14:2135.

[11] Diankov G, Liang CT, Amet F, Gallagher P, Lee M, Bestwick AJ, et al. Robust fractional quantum Hall effect in the $\mathrm{N}=2$ Landau level in bilayer graphene. Nature Comm. 2016;7:13908.

[12] Kou A, Feldman BE, Levin AJ, Halperin BI, Watanabe K, Taniguchi T, et al. Electron-hole asymmetric integer and fractional quantum Hall effect in bilayer graphene. Science. 2014;345(6192):55-57.

[13] Maher P, Wang L, Gao Y, Forsythe C, Taniguchi T, Watanabe K, et al. Tunable fractional quantum Hall phases in bilayer graphene. Science. 2014;345(6192):61-64. 
[14] Kim Y, Lee DS, Jung S, SkÃąkalovÃą V, Taniguchi T, Watanabe K, et al. Fractional Quantum Hall states in bilayer graphene probed by transconductance fluctuations. Nano Lett. 2015;15(11):7445-7451.

[15] Dean CR, Young AF, Cadden-Zimansky P, Wang L, Ren H, Watanabe K, et al. Multicomponent fractional quantum Hall effect in graphene. Nature Physics. 2011;7:693.

[16] Amet F, Bestwick AJ, Williams JR, Balicas L, Watanabe K, Taniguchi T, et al. Composite fermions and broken symmetries in graphene. Nature Comm. 2015;6:5838.

[17] Feldman BE, Krauss B, Smet JH, Yacoby A. Unconventional sequence of fractional quantum Hall states in suspended graphene. Science. 2012;337:1196.

[18] Feldman BE, Levin AJ, Krauss B, Abanin DA, Halperin BI, Smet JH, et al. Fractional quantum Hall phase transitions and four-flux states in graphene. Phys Rev Lett. 2013;111:076802.

[19] Castro Neto AH, Guinea F, Peres NMR, Novoselov KS, Geim AK. The electronic properties of graphene. Rev Mod Phys. 2009;81:109.

[20] Zhang Y, Jiang Z, Small JP, Purewal MS, Tan YW, Fazlollahi M, et al. Landau-level splitting in graphene in high magnetic fields. Phys Rev Lett. 2006;96:136806.

[21] Jacak J, Jacak L. Recovery of Laughlin correlations with cyclotron braids. Europhysics Lett. 2010;92:60002.

[22] Jacak J, Jacak L. The commensurability condition and fractional quantum Hall effect hierarchy in higher Landau levels. JETP Lett. 2015;102:19-25.

[23] Łydżba P, Jacak L, Jacak J. Hierarchy of fillings for the FQHE in monolayer graphene. Sci Rep (Nature). 2015;5:14287.

[24] Jacak J, Jacak L. Difference in hierarchy of FQHE between monolayer and bilayer graphene. Phys Lett A. 2015;379:2130.

[25] Eisenstein JP, Lilly MP, Cooper KB, Pfeiffer LN, West KW. New physics in high Landau levels. Physica E. 2000;6:29.

[26] Dolev M, Gross Y, Sabo R, Gurman I, Heiblum M, Umansky V, et al. Characterizing neutral modes of fractional states in the second Landau level. Phys Rev Lett. 2011;107:036805.

[27] Willett RL. The quantum Hall effect at 5/2 filling factor. Rep Prog Phys. 2013;76:076501.

[28] Goerbig MO. Electronic properties of graphene in a strong magnetic field. Rev Mod Phys. 2011;83:1193.

[29] McCann E, Falko VI. Landau-level degeneracy and quantum Hall effect in a graphite bilayer. Phys Rev Lett. 2006;96:086805.

[30] Papić Z, Abanin DA. Topological phases in the zeroth Landau level of bilayer graphene. Phys Rev Lett. 2014;112:046602.

[31] Du X, Skachko I, Duerr F, Luican A, Andrei EY. Fractional quantum Hall effect and insulating phase of Dirac electrons in graphene. Nature. 2009;462:192. 
[32] Bolotin KI, Ghahari F, Shulman MD, Störmer HL, Kim P. Observation of the fractional quantum Hall effect in graphene. Nature. 2009;462:196.

[33] Abanin DA, Skachko I, Du X, Andrei EY, Levitov LS. Fractional quantum Hall effect in suspended graphene: transport coefficients and electron interaction strength. Phys Rev B. 2010;81:115410.

[34] Skachko I, Du X, Duerr F, Luican A, Abanin DA, Levitov LS, et al. Fractional quantum Hall effect in suspended graphene probed with two-terminal measurements. Phil Trans $R$ Soc A. 2010;368:5403.

[35] Papić Z, Goerbig MO, Regnault N. Atypical fractional quantum Hall effect in graphene at fillig factor 1/3. Phys Rev Lett. 2010;105:176802.

[36] Novoselov KS, Geim AK, Morozov SV, Jiang D, Katsnelson MI, Grigorieva IV, et al. Twodimensional gas of massles Dirac fermions in graphene. Nature. 2005;438:197.

[37] Zhang Y, Tan YW, Störmer HL, Kim F. Experimental observation of the quantum Hall effect and Berry's phase in graphene. Nature. 2005;438:201.

[38] Yannouleas C, Romanovsky I, Landman U. Edge and bulk components of lowest-Landau-level orbitals, correlated fractional quantum Hall effect incompressible states, and insulating behavior of finite graphene samples. Phys Rev B. 2010;82:125419.

[39] Dahal HP, Joglekar YN, Bedell KS, Balatsky AV. Absence of Wigner crystallization in graphene. Phys Rev B. 2006;74:233405.

[40] Pfeiffer L, West KW. The role of MBE in recent quantum Hall effect physics discoveries. Physica E. 2003;20:57.

[41] Bolotin KI, Sikes KJ, Jiang Z, Klima M, Fudenberg G, Hone J, et al. Ultrahigh electron mobility in suspended graphene. Solid State Comm. 2008;146:351.

[42] Suen YW, Engel LW, Santos MB, Shayegan M, Tsui DC. Observation of a $v=1 / 2$ fractional quantum Hall state in a double-layer electron system. Phys Rev Lett. 1992;68:1379.

[43] Eisenstein JP, Boebinger GS, Pfeiffer LN, West KW, He S. New fractional quantum Hall state in double-layer two-dimensional electron systems. Phys Rev Lett. 1992;68:1383. 